\title{
Programa de Residência em Medicina de Família e Comunidade da UERJ: uma perspectiva histórica
}

\author{
Family and Community Medicine Residency Program of the \\ State University of Rio de Janeiro: a historical perspective
}

Ricardo Donato Rodrigues*

\begin{abstract}
Resumo
O Programa de Residência em Medicina de Família e Comunidade da Faculdade de Ciências Médicas da UERJ é um dos pioneiros desta especialidade no Brasil - foi criado, junto com dois outros, em 1976. Este artigo apresenta o cenário político da época, inclusive quanto às dificuldades para implantar um programa centrado em uma perspectiva inovadora no campo da saúde. Apresenta, de forma sucinta, o marco teórico-conceitual e os eixos de estruturação do PRMFC da UERJ, contrapostos a uma concepção simplista sobre Atenção Primária à Saúde (APS) e formação profissional nesta área que ainda predomina em alguns círculos, realçando que concepções simplistas não fazem justiça à complexidade das questões de saúde que se apresentam neste nível de atenção. Ressalta que, para promover saúde, prevenir a ocorrência de doenças evitáveis de complicações precoces de doenças instaladas que carregam o risco de comprometer a autonomia, provocar invalidez ou morte prematura, é necessário desenvolver competências específicas, razão de ser de um Programa de Residência em Medicina de Família e Comunidade e da própria especialidade. Por fim, discorre sobre os campos de prática e aponta perspectivas, sem deixar de assinalar dificuldades institucionais, em especial aquelas relativas aos cenários de treinamento, ressaltando, entretanto, que o programa Saúde na Vila - implantado no entorno do complexo docenteassistencial da UERJ - tem constituído uma alternativa alentadora.
\end{abstract}

\begin{abstract}
The Family and Community Medicine Residency Program of the Medical Course of the Rio de Janeiro State University is a forerunner of this specialty in Brazil. The program was established in 1976, together with two other programs. This article portrays the political scenario of that time describing the struggles to start a program focused on an innovative perspective. It provides an idea of the theoretical and conceptual framework and of the structure of the Family and Community Medicine Residency Program of the State University of Rio de Janeiro, in contraposition to a simplistic concept of Primary Care and the qualification of professionals in this area. Such simplistic
\end{abstract}

Palavras-chave: Medicina de Família e

Comunidade; Residência Médica; Atenção

Primária à Saúde.
Key Words: Family Practice; Internship and

Residency; Primary Healthcare.

*Médico, Doutor em Saúde Coletiva, Professor Adjunto, Departamento de Medicina Integral, Familiar e Comunitária da Faculdade de Ciências Médicas da Universidade do Estado do Rio de Janeiro, Rio de Janeiro, Brasil. 
conceptions however, though still prevailing in some circles, are unfit for facing the complexity of the health issues at this care level. It is stressed that for promoting health it is necessary to develop specific competences, prevent diseases and avoid precocious effects of existing diseases capable of compromising the individual's autonomy and of causing disablement or sudden death. This is the reason of being of the Family and Community Medicine Residency Program and of the specialty itself. Finally the article addresses necessary practical actions, among others with respect to institutional difficulties especially those concerning training facilities. On the other hand, the program "Saúde na Vila" - a program for integral healthcare delivery maintained in the neighborboods of the University Hospital - is emphasized as an extremely encouraging alternative.

\section{Primórdios}

O Programa de Residência em Medicina de Família e Comunidade (RMFC) da Faculdade de Ciências Médicas/ UERJ (FCM/UERJ) foi criado em 1976, ainda sob a denominação de Residência em Medicina Integral. Neste mesmo ano foram instituídos, em Murialdo, Porto Alegre, e Vitória de Santo Antão, na região metropolitana de Recife, dois outros Programas de Residência em Medicina Geral Comunitária (RMGC), denominação que se tornou obrigatória a partir de 1981, com a regulamentação dos Programas de Residência Médica no país pela Comissão Nacional de Residência Médica (CNRM). Finalmente, em 2002, por proposta da SBMFC, a CNRM substituiu a denominação original por Medicina de Família e Comunidade'.

Esses três programas, pioneiros, de residência foram instituídos, portanto, dois anos antes da histórica Conferência Internacional de Alma-Ata, que, sob inspiração de Halfdan Mahler, então diretor executivo da Organização Mundial de Saúde, consagrou a meta "Saúde Para Todos no Ano 2000" e elegeu os "Cuidados Primários de Saúde" como estratégia privilegiada para alcançá-la². Nesta época, entretanto, o setor saúde no Brasil avançava em outra direção ${ }^{3}$.

Foi necessário enfrentar poderosos obstáculos para implantar um programa de residência que visava à formação de especialistas a partir de uma racionalidade médica inovadora, capaz de torná-los protagonistas de um processo de mudança do modelo assistencial adotado neste país, cuja hegemonia, entretanto, estende-se até os dias atuais.

Os anseios por mudanças tanto no plano da educação médica quanto da prática médica vinham de longe. Em 1968 - ano em que a juventude, unida pelo sonho de ver o nascimento de dias melhores para a humanidade, tomou conta das ruas do mundo, inclusive no Rio -, um grupo de alunos da FCM-UERJ, desafiando a brutalidade policialmilitar instaurada no país pelo golpe de 64, iniciou um estudo sobre o ensino médico na área das Ciências Médicas, por meio da aplicação de um questionário aos alunos do $2^{\circ}$ ao $6^{\circ}$ ano. Essa iniciativa, desencadeada a partir de uma visão que considerava o ensino médico na FCM (como de resto, no país) elitista, voltado para o atendimento hospitalar de doenças raras, desconsiderando as necessidades de saúde da maioria da população, visava à "alimentação" de um processo de discussão capaz de gerar subsídios para a construção de uma proposta discente de reforma curricular.

A área das Ciências Médicas vivia dias agitados, a ditadura não dava trégua ao movimento estudantil e as forças da repressão "chegavam com tudo" às portas da escola - um estudante, baleado na cabeça, morreu no centro cirúrgico do Hospital Universitário Pedro Ernesto (então, Hospital de Clínicas da UEG). A violência abortou a constituição de Comissões Paritárias, formadas por professores e alunos, que levariam adiante o processo de reforma curricular ${ }^{4}$. Entretanto, ela não conseguiu deter o desenvolvimento do Internato Geral Rotativo, destinado ao treinamento prático dos alunos dos últimos períodos nas chamadas áreas básicas, logo adotado pela FCM; tampouco, conseguiu desmantelar o recém-criado Serviço Ambulatorial de Medicina Integral (AMI).

Afinal, o mundo já vinha dando mostras de insatisfação com os modelos da educação e os resultados das práticas no âmbito da saúde; sobretudo, uma insatisfação com os elevados custos da assistência médico-hospitalar em decorrência do acelerado processo de fracionamento da Medicina em especialidades. Assim, pouco a pouco foram surgindo condições favoráveis ao florescimento de experiências inovadoras, tanto relacionadas ao desenvolvimento da 
Medicina Preventiva quanto da Medicina Comunitária e Medicina Integral ${ }^{5}$.

\section{Do Serviço de Medicina Integral ao Programa de} Residência em Medicina de Família e Comunidade

A criação do AMI com o objetivo de propiciar treinamento prático em medicina ambulatorial sob supervisão representava o primeiro passo de uma longa jornada, pois ainda havia uma distância considerável entre intenção e gesto. No Brasil, a exemplo de tantos países, a atenção médica seguia os ditames do modelo biomédico e o ensino seguia rezando a cartilha flexneriana. Assim, este ambulatório do Pedro Ernesto, não raro, assemelhava-se, nesta época, a uma ante-sala das enfermarias, selecionando casos (ditos) interessantes para internação de acordo com as necessidades de ensino. Mantinha uma clientela pequena e cativa, com agravos crônicos, submetida às regras de uma medicina cientificista.

Este embrionário projeto de Medicina Integral não parecia suficiente para suplantar uma visão de prática médica hospitalocêntrica centrada em uma concepção biotecnológica. As atenções continuavam dirigidas fortemente para o diagnóstico e tratamento das doenças. Assim, persistiam existindo velhos formulários de muitas páginas para o registro exaustivo das queixas clínicas, da história da doença atual, da anamnese dirigida e inventário sistematizado dos sistemas orgânicos. Enquanto isso, o interrogatório nos terrenos sociodemográfico, familiar e epidemiológico comprometia-se, basicamente, com a formulação de um dignóstico etiopatogênico. O exame físico detalhado e exames laboratoriais em profusão, solicitados a título de rotina, serviam para reforçar o foco na doença ${ }^{6}$.

Tais práticas constituíam uma espécie de paradigma de excelência para a rede assistencial e para a educação médica. Para alguns, ainda hoje é assim. Entretanto, a ineficiência desse modelo, sua baixa capacidade resolutiva, o uso abusivo de medicinas e hospitalizações, os acidentes iatrogênicos, os custos explosivos e tantas outras distorções acabavam engrossando o sentimento de insatisfação que tomava conta de usuários, estudantes e setores profissionais.
Os próprios hospitais universitários sofriam as conseqüências nefastas de tais práticas. Movidos pela escassez de recursos puseram-se atrás de novas fontes de financiamento ${ }^{7,8}$.

Nesta ocasião, na tentativa de sanar problemas decorrentes do desmantelamento do milagre econômico brasileiro, o Instituto Nacional de Previdência Social (INPS) estabeleceu, dentre outras medidas, convênios ampliados com o setor público, particularmente com os hospitais universitários. O Pedro Ernesto foi o segundo hospital universitário do país a assinar, no final de 1974, esta modalidade inovadora de convênio com o INPS. Foi exponencial o crescimento da demanda hospitalar, inclusive da demanda ambulatorial, em decorrência deste convênio.

Para fazer frente a esta nova conjuntura, inclusive a esta nova demanda, crescente e diversificada, a alternativa da instituição, apoiada por atores engajados nas iniciativas prévias de reforma docente-assistencial, foi reestruturar o AMI a partir de uma visão holística da tríade saúde-doençacuidado?. Nesse contexto, implantou-se finalmente o Programa de Residência de Medicina de Família e Comunidade na UERJ.

Estruturada de acordo com um regime eminentemente extra-hospitalar de treinamento, esse programa de Residência da FCM/UERJ tinha por finalidade formar especialistas em Medicina de Família e Comunidade visando a contribuir, deste modo, para viabilizar a implantação e potencializar o desenvolvimento de projetos que se inscrevem no âmbito da Atenção Primária à Saúde. Para tanto, esperava-se que este profissional tivesse elevada qualificação clínica, capacidade de planejar, implementar e avaliar as ações de saúde no nível de sua área de atuação, além de realizar estudos/pesquisas com base na população a qual assistia, como forma de adequar e aperfeiçoar a sua prática.

Desde o início, portanto, o PRMFC visava à formação de especialistas que iriam atuar em um campo de práticas que emergiu e se organizou com o propósito de fazer frente aos graves problemas experimentados pela humanidade em termos de atenção à saúde. Isso é, objetivava - e ainda o faz - formar especialistas que iriam atuar em um campo que se organizou em contraposição às distorções 
do atual modelo assistencial hospitalocêntrico, particularmente no que diz respeito à crescente especialização biotécnica dos cuidados de saúde e ao consumo abusivo e irracional de medicinas.

Em vista dessas considerações, esperava-se, desde o início, que o egresso deste PRMFC se convertesse antes de tudo em agente de mudança no campo da saúde, recusando-se a assumir um papel de mero prestador de serviços subserviente aos interesses do mercado, sem compromisso maior com a saúde individual e com a saúde coletiva. Esperava-se que este egresso não dominasse apenas a teoria das doenças, mas fosse capaz de entender e lidar de forma responsável e competente com a saúde das pessoas, com a saúde das famílias, da sociedade, enfim, com a vida humana. Esperava-se que o egresso soubesse valorizar a relação médico-paciente e entendesse a importância do trabalho em equipe e da participação individual e comunitária nas questões de saúde. Era esperado também que desenvolvesse competências no campo da resiliência, isto é, da capacidade de superação, dessa totalidade biopsicossocial que é o ser humano ${ }^{10}$.

\section{Obstáculos encontrados no caminho}

É óbvio que um PRMFC com as características sumariamente apresentadas acima depara-se com numerosos problemas e enfrenta sérias dificuldades. Mas não são obstáculos conjugados somente no passado. Ao contrário, são recorrentes e não se deixam de projetar no presente. Efetivamente, não parece difícil imaginar o que se passa com um programa em nível de pós-graduação que não está em sintonia com os programas hegemônicos, seja em termos docentes, isto é, em termos do ensino, pesquisa e produção de conhecimento no campo médico, seja em termos da prática médica. Em outras palavras, não parece difícil imaginar os problemas que afetam um programa docente-assistencial que se desenvolvia até pouco tempo às margens das políticas governamentais das áreas da educação e da saúde, um programa periférico, mas viável enquanto, lá nos confins do sistema de saúde, serve a alguns interesses pragmáticos das instituições e seus dirigentes.
Até hoje, duas questões de fundo sempre aparecem na literatura e no círculo de debates quando se pretende questionar a necessidade de instituir programas de residência na área da Medicina de Família e Comunidade certamente, não faltaram no cenário da Faculdade de Ciências Médicas. A primeira, cujas variações semânticas não conseguem disfarçar o tom pejorativo que vez por outra acompanha o questionamento, argumenta que não seria necessário existir um programa de pós-graduação nesta área, pelo menos na modalidade de residência médica, em face ao conceito de "terminalidade" do ensino de graduação em Medicina.

A equipe do antigo Ambulatório de Medicina Integral, hoje transformado em Departamento de Medicina Integral, Familiar e Comunitária, sempre esposou uma tese sobre a "terminalidade" do ensino de graduação, a qual significa que a Escola Médica tem a responsabilidade de formar, em seis anos, um profissional com conhecimentos suficientes para atuar em qualquer ramo da Medicina, desde que inserido em uma equipe de trabalho, compartilhando sua prática especializada com colegas mais experientes.

De acordo com esta tese esposada pela equipe do Departamento de Medicina Integral, Familiar e Comunitária da FCM/UERJ, a Escola Médica tem, portanto, a responsabilidade de formar um profissional altamente qualificado, ainda que recém-formado - vale dizer, com menos experiência. Neste sentido, entende que tal recémformado deverá passar por um período de amadurecimento, experimentará um período de transição, tanto mais produtivo e menos traumático, para si e para outros, à medida que for acolhido em um ambiente organizado para tal fim.

A experiência nacional e internacional tem revelado que a Residência Médica cumpre este papel com a vantagem de expor o recém-formado a programas regulamentados que, além da prática em serviço sob supervisão, exigem a verticalização do conhecimento teórico nas respectivas especialidades médicas; quando não, a produção de novos conhecimentos. Mais ainda, como não são estabelecidas relações trabalhistas típicas entre residentes e instituição 
fomentadora do programa, criam-se condições especiais que permitem avaliações críticas, independentes da influência direta de gerentes e patronato, a propósito das políticas e programas assistenciais, "descortinando", dessa maneira, novos horizontes favoráveis ao aperfeiçoamento do próprio sistema de atenção à saúde.

Uma segunda linha de argumentação, que também não faltou no cenário da UERJ, sustenta que não há necessidade de instituir programas de residência no âmbito da Atenção Primária à Saúde (APS), como o PRMFC, sob a alegação que, neste nível do sistema de saúde, são atendidas condições ou problemas simples de saúde. Nessa concepção, os problemas mais complexos seriam referidos para os centros de Atenção Secundária ou Terciária, isto é, centros que, nesta mesma lógica, são considerados mais especializados de um sistema hierarquizado em níveis crescentes de complexidade.

Aqui, os problemas da argumentação provocam múltiplas contestações. Uma expressão corrente, que ecoa no Departamento de Medicina Integral e Familiar da FCM/ UERJ desde a década de 1970, deve-se a Abraam Sonis e José Maria Paganini, autores argentinos que sustentavam que tal assertiva confundia APS com Atenção Primitiva, isto é, com "uma medicina de segunda classe para cidadãos de segunda classe prestada por pessoal de segunda classe" ${ }^{11}$.

A equipe de preceptores do PRMFC da FCM/ UERJ tem insistido ao longo dos anos que aquela concepção simplista não faz justiça à complexidade das questões de saúde que se apresentam no campo da Atenção Primária. Além da responsabilidade de fazer frente a necessidades de saúde de várias ordens, este nível do sistema tem um compromisso com a saúde de todos. A APS tem, entre outras, a função de promover saúde; prevenir a ocorrência de doenças evitáveis; evitar a ocorrência de complicações precoces de doenças instaladas que carregam o risco de comprometer a autonomia, provocar invalidez ou mesmo a morte prematura das pessoas. Para tanto, é necessário desenvolver competências específicas, razão de ser de um Programa de Residência em Medicina de Família e Comunidade e desta própria especialidade.
Vale aqui mencionar alguns pontos deste PMFC, relacionados à complexidade envolvida na APS, que vêm merecendo uma atenção muito especial da equipe do Departamento. O primeiro diz respeito à formação de Grupo Balint com os residentes de $1^{\circ}$ ano, realizado semanalmente até o final deste período da residência. $\mathrm{O}$ segundo ponto é o treinamento teórico-prático para o Trabalho com Grupos que é realizado durante todo o segundo ano da residência. $\mathrm{O}$ terceiro, que tem merecido uma atenção especial e ocupa os dois anos da residência, diz respeito à Abordagem Familiar compreendendo um momento teórico semanal e atividades práticas, incluindo interconsultas e consultas conjuntas com terapeuta de família.

O quarto ponto é o treinamento teórico-prático em Saúde Mental na APS também com interconsultas e consultas conjuntas com a equipe da Psicologia Médica da FCM. O quinto ponto é um Programa de Saúde Escolar, voltado para a promoção do autocuidado, da resiliência e da qualidade de vida de estudantes, professores e funcionários desta comunidade escolar. O programa é implementado a partir de um diagnóstico de saúde e do interesse revelado por esta comunidade. Após o estabelecimento de vínculos e relações de confiança, a equipe implementa ações de saúde, especialmente no plano da educação em saúde escolar, com a participação ativa tanto de residentes de Medicina de Família e Comunidade quanto de estudantes de graduação.

Todos esses pontos são articulados e reforçados no contexto do Programa Saúde na Vila, que é um cenário de práticas docente-assistenciais no campo da saúde da família constituído no entorno do Hospital Universitário Pedro Ernesto e hoje representa um dos eixos estruturantes do PRMFC.

Por último, vale fazer menção particular à Clínica do Médico de Família, tema que até hoje tem sido objeto de estudos e discussões.

O Departamento de Medicina Integral, Familiar e Comunitária da UERJ entende que a clínica compreende a prestação de cuidados de saúde a pessoas cuja primeira etapa é o estabelecimento de um diagnóstico. Enquanto os 
especialistas focais da atenção secundária e da atenção terciária priorizam os diagnósticos da doença e das alterações fisiopatológicas exibidas por cada doente particular, os especialistas em Medicina de Família e Comunidade inseridos na APS, postos frente a frente com um doente, priorizam o diagnóstico da doença, o respectivo leque de condicionantes envolvidos e a teia por eles tecida em cada situação particular.

Parece óbvio que, frente a um paciente com dor no peito, por exemplo, ambos possam estabelecer um diagnóstico de infarto agudo do miocárdio e tomar as primeiras medidas terapêuticas indicadas. Entretanto, depois de um exame clínico objetivo, os cardiologistas certamente irão mobilizar recursos subespecializados para, em primeiro lugar, identificar alterações anatômicas e os mecanismos fisiopatológicos envolvidos, e, logo adiante, definir a estratégia terapêutica mais indicada. Quando for o caso, podem mobilizar um outro contingente de especialistas para realizar novos procedimentos no campo do diagnóstico ou da terapêutica. Ou seja, empreendem uma prática à luz do paradigma biomédico que tem como eixo estruturante a teoria das doenças então concebidas como um conjunto de sinais e sintomas conseqüentes a alterações anatômicas e fisiopatológicas provocadas na intimidade tecidual pela ação de agentes causais específicos.

Já o especialista em Medicina de Família e Comunidade, iluminado pelo paradigma da integralidade biopsicossocial, que concebe o fenômeno saúde-doença como um processo condicionado por um emaranhado de variáveis biológicas e psicossociais articuladas por uma teia de relações, sabe que é necessário exercer uma clínica que possibilite o diagnóstico das condições que favorecem ou causam prejuízo à saúde do paciente e, por conseqüência, viabilize a prática de um cuidado integral. Ou seja, em Medicina de Família e Comunidade, não basta diagnosticar a doença para instituir um cuidado adequado. Para isso, também é necessário diagnosticar as condições que interferem no seu curso, sejam condições internas de ordem biopsíquica, sejam condições externas, inclusive as redes sociais de apoio e facilidades de acesso a serviços de saúde de qualidade.
Em face das questões apresentadas até aqui neste texto, o Departamento de Medicina Integral da FCM/ UERJ considera que a formação de especialistas em Medicina de Família e Comunidade não é, definitivamente, uma questão trivial. Ao contrário de arremedo, é uma medicina de alta complexidade, cuja dimensão clínica, centrada no paradigma da integralidade, é de natureza biopsicossocial.

\section{Cenários de Prática e Perspectivas}

O Programa de Residência em Medicina de Família e Comunidade foi instituído em 1976, portanto, dois anos antes da Conferência de Alma-Ata e 18 anos antes da adoção, pelo Ministério da Saúde, do Programa Saúde da Familia, hoje, Estratégia Saúde da Família (ESF). Nesta época, como se sabe, o Sistema de Saúde no Brasil, como de resto, na maior parte do mundo, caracterizava-se por um modelo assistencial hospitalocêntico, organizado em torno de grandes centros especializados de diagnóstico e tratamento, inclusive hospitais de todo tipo e porte. A Saúde Pública ficava por conta de um Ministério da Saúde sempre às voltas com uma dotação orçamentária insuficiente, quando não, irrisória. Assim sendo, boa parte da Atenção Primária ficava por conta do improviso.

Neste contexto de desorganização da APS no Rio de Janeiro e em outras cidades do planeta, o Programa de Residência nos primeiros anos era subdividido em dois grandes módulos. Um, eminentemente clínico e de natureza teórico-prática, concentrava-se em consultórios da Unidade de Pacientes Externos do Hospital Universitário Pedro Ernesto, situado em prédio anexo ao mesmo, no Campus Biomédico da UERJ, bairro de Vila Isabel. O outro módulo, dominantemente voltado para a Medicina Comunitária, localizava-se no bairro de Austin, município de Nova Iguaçu, onde a UERJ, com apoio da Fundação Kellog e em parceria com a Secretaria de Saúde daquele município, desenvolvia um programa de Atenção Primária. Apesar dos inconvenientes, sobretudo no que diz respeito às distâncias entre os dois cenários, foi com este desenho que se formaram as primeiras turmas. 
Alguns anos depois, com o esgotamento do apoio da Kellog e da parceria com o município de Nova Iguaçu, por iniciativa conjunta da Direção Geral do Hospital Universitário Pedro Ernesto e da Coordenação do Programa de Residência, foi implantado um programa de APS na comunidade do Borel, que se tornou um novo cenário de prática da Residência. Foi constituída uma equipe própria, multidisciplinar, cuja coordenação foi exercida inicialmente por um médico e, depois, por uma enfermeira. Durante anos foi possível desenvolver um trabalho compartilhado e proveitoso, bem aceito pelos residentes e pela comunidade. Apesar de a unidade localizar-se bem no centro de uma comunidade, em uma região considerada de risco, as equipes tinham trânsito livre, sendo alertadas, quando necessário, pela própria associação de moradores. Mas, um dia, aconteceu uma ação inesperada da polícia civil e o veículo que transportava uma equipe ficou sob fogo cruzado, desencadeando uma situação inaceitável de convivência.

Aos poucos se foi organizando um novo programa, ainda que incipiente, de Medicina de Família e Comunidade no entorno do Hospital Universitário. Com a constituição do Pólo de Formação, Capacitação e Educação Permanente de Pessoal para a Saúde da Família e a organização e implantação das primeiras equipes de Saúde da Família pela Secretaria Municipal de Saúde, criou-se grande expectativa no sentido de redesenhar o Programa de Residência e deslocar o treinamento dos residentes para as novas Unidades Básicas da Secretaria Municipal do Rio de Janeiro.

Entretanto, a implantação muito lenta da ESF neste município e a localização das unidades em conhecidas áreas conflagradas da cidade, serviram para arrefecer o ímpeto inicial do Departamento que, desde então, vem ampliando um Programa de Medicina de Família e Comunidade, iniciado anos antes no entorno do campus biomédico da UERJ. Chamado de Saúde na Vila este programa compreende sete microáreas cuja população totaliza cerca de 80 mil pessoas.

As atuais perspectivas parecem mais animadoras. Tudo faz crer que a intenção do Departamento de Medicina de Família e Comunidade de retomar um projeto de integração academia-serviço, fortalecer e tornar o Saúde na
Vila um programa de interesse interinstitucional, visando ao aperfeiçoamento da ESF no Rio de Janeiro, poderá ser bem sucedida. Afinal, as Secretarias Municipais e a Secretaria de Estado da Saúde e Defesa Civil vêm reconhecendo, nos últimos tempos, a importância da capacitação profissional e da formação de especialistas em Medicina de Família e Comunidade para promover tal aperfeiçoamento. Inclusive, vêm participando e concedendo apoio à realização de congressos e outros eventos científicos relacionados à APS, sejam promovidos pela academia ou pela Sociedade Brasileira de Medicina de Família e Comunidade e respectiva regional.

Seja como for, todos sabem que a saúde no Rio precisa mudar. E as instâncias gestoras da Atenção Básica desta cidade e desse estado sabem que o Programa de Residência em Medicina de Família e Comunidade tem, pelo menos, 32 anos de história para contar.

\section{Referências}

1. Falk JW. A Medicina de Família e Comunidade e Sua Entidade Nacional: Histórico e Perspectivas. Rev Bras de Med de Fam e Com. Abr. 2004; 1 (1): 5-10.

2. UNICEF. Cuidados Primários de Saúde. Brasília (DF): UNICEF; 1979. 64p.

3. Mello CG. O Sistema de Saúde em Crise. São Paulo, Rio de Janeiro: Cebes, Hucitec; 1981. 205p.

4. Daflon F. Título Provisório: O Movimento Estudantil nas Ciências Médicas. São Paulo: Quilombo; 1980. 103p. 5. Silva Jr AG. Modelos Tecnoassistenciais em Saúde: o debate no campo da saúde coletiva. São Paulo: HUCITEC; 1998. 143P. 6. Landmann J. Evitando a Saúde e Promovendo a Doença. Rio de Janeiro: Achiamé; 1982. 187p.

7. Brasil. Ministério da Educação e Cultura. Documentos do Ensino Médico. Brasília(DF): MEC; 1989. 204p.

8. Mello CG. O Sistema de Saúde em Crise. São Paulo, Rio de Janeiro: Cebes, Hucitec; 1981. 205p.

9. Noronha JC; Oliveira JA; Rodrigues RD; Landmann J. Transformação de um Ambulatório de Medicina Integral com Vistas a um Programa de Atenção Médica Primária. Rev Saúde Públ. 1977; 11: 429-43.

10. Rodrigues RD. Notas sobre um Programa de Residência 
em Ambulatório. XXV Congresso Brasileiro de Homeopatia; 2002. Rio de Janeiro: Sociedade Brasileira de Homeopatia; 2002. p.201-4.

11. Sonis A; Paganini JM. Medicina Sanitária y Administración de Salud. Tomo II. Buenos Aires: El Ateneo; 1976. 341p.

\section{Endereço para Correspondência:}

Rua 28 de setembro, sala 605 ,

Rio de Janeiro RJ

CEP: 20.551-031

\section{Endereço Eletrônico:}

ricardodonato@terra.com.br 